\title{
Identical elements model of arithmetic memory: Extension to addition and subtraction
}

\author{
JAMIE I. D. CAMPBELL \\ University of Saskatchewan, Saskatoon, Saskatchewan, Canada \\ and \\ SHANNON FUCHS-LACELLE and THOMAS L. PHENIX \\ University of Regina, Regina, Saskatchewan, Canada
}

\begin{abstract}
The identical elements (IE) model of arithmetic fact representation (Rickard, 2005; Rickard \& Bourne, 1996) was developed and tested with multiplication and division. In Experiment 1, we demonstrated that the model also applies to addition and subtraction by examining transfer of response time (RT) savings between prime and probe problems tested in the same block of trials. As is predicted by the IE model, there were equivalent probe RT savings for addition with identical repetition (prime $6+$ $9 \rightarrow$ probe $6+9)$ or an order change $(9+6 \rightarrow 6+9)$, but much greater savings for subtraction with identical repetition $(15-6 \rightarrow 15-6)$ than with an order change $(15-9 \rightarrow 15-6)$, and no savings with an operation change $(15-9 \rightarrow 6+9$ or $6+9 \rightarrow 15-6)$. In Experiment 2 , we examined transfer in simple multiplication and division and demonstrated symmetrical transfer between operations. Cross-operation RT savings were eliminated, however, when the RT analysis included only trials on which both the prime and the probe problems were reportedly solved by direct retrieval. An IE model extended to accommodate savings associated with procedural strategies provides a coherent account of facilitative transfer effects in simple arithmetic.
\end{abstract}

The cognitive processes that mediate simple arithmetic skills have been the focus of extensive experimental research over the last 30 years (Ashcraft, 1992, 1995; Zbrodoff \& Logan, 2005). This research was an attempt to identify the memory processes and procedural strategies that underlie performance of basic arithmetic problems, such as $2+3=5$ and $6 \times 7=42$. The work is motivated both by the importance of understanding the nature of this fundamental intellectual skill and by the fact that cognitive arithmetic provides a rich experimental domain for studying more general theoretical issues in cognitive science (Butterworth, 1999; Dehaene, 1997).

This article reports two experiments that were performed to test the predictions of a prominent theory of arithmetic fact representation: the identical elements (IE) model (Rickard, 2005; Rickard \& Bourne, 1996; Rickard, Healy, \& Bourne, 1994). According to the IE model, there is a single long-term memory node for problems consisting of the same numerical elements (i.e., operands and answer), regardless of operand order. For example, each multiplication node specifies the two operands, the opera-

This research was supported by a grant from the Natural Sciences and Engineering Research Council of Canada. We thank Tim Rickard and two anonymous reviewers for very helpful feedback on a previous version of the manuscript. Address correspondence to J. I. D. Campbell, Department of Psychology, University of Saskatchewan, 9 Campus Drive, Saskatoon, SK, S7N 5A5 Canada (e-mail: jamie.campbell@usask.ca).

Note-This article was accepted by the previous editorial team, when Colin M. MacLeod was Editor. tion, and the product (e.g., $6,8, \times \rightarrow 48$ ) and is accessed by either operand order $(6 \times 8$ or $8 \times 6)$. In contrast, inverse problems that present different operands and have different answers access different nodes. Thus, inverse division problems (e.g., $48 \div 8$ and $48 \div 6$ ) are represented by different nodes $[(48,8, \div \rightarrow 6)$ and $(48,6, \div \rightarrow 8)]$.

Given these assumptions, practice should strengthen only the node corresponding to the practiced problem. Consequently, positive transfer of savings should occur equally between the two orders of multiplication problems, but there should be no transfer between the two orders of division problems and no transfer between inverse multiplication and division problems. Rickard and Bourne (1996) gave adults extensive practice on subsets of simple multiplication and division problems and then tested performance on the same problems (identical repetition), problems with the operand order reversed (order change), inverse problems with the other operation (operation change), and unpracticed control problems. Transfer of practice (response time [RT] savings) in the order change condition for multiplication (e.g., practice, $4 \times 3$; test, $3 \times 4$ ) was practically equivalent to identical repetition (practice, $3 \times 4$; test, $3 \times 4$ ), which supports the assumption that a common representation mediates both orders (see Verguts \& Fias, 2005). They found no transfer of savings between the two orders of division problems (practice, $56 \div 7=8$; test, $56 \div 8=7$ ) and little evidence that practice transferred in either direction between corresponding division and multiplication problems (practice, $7 \times 8=56$, and test, $56 \div 8=7$; or practice, $56 \div 8=$ 7 , and test, $7 \times 8=56$ ). 
Rickard et al.'s (1994; Rickard \& Bourne, 1996) data supported the IE model (see also Cipolotti \& de Lacy Costello, 1995; McCloskey, Aliminosa, \& Sokol, 1991), but subsequent transfer experiments testing simple multiplication and division suggested that the IE model is not the whole story. Campbell (1999) used a transfer paradigm that measured the effects of a single prime problem on a probe problem tested four to six trials later in the same block. Multiplication and division prime-probe pairs corresponded to identical repetition, order change, and operation change transfer conditions. As is predicted by the IE model, RT savings for division were larger with identical repetition (e.g., prime, $63 \div 7$; probe, $63 \div 7$ ) than with an order change $(63 \div 9 \rightarrow 63 \div 7)$, whereas identical $(7 \times 9$ $\rightarrow 7 \times 9)$ and commuted $(9 \times 7 \rightarrow 7 \times 9)$ multiplication problems produced equivalent transfer. Nonetheless, there were transfer effects not predicted by the model, including a significant transfer of savings between the two orders of division problems. In addition, the larger, more difficult division problems presented substantial RT savings in the multiplication-to-division transfer condition (e.g., prime, $7 \times 9$; probe, $63 \div 7$ ).

Campbell (1999) proposed that transfer between orderreversed division problems and between multiplication and division could be reconciled with the IE model by distinguishing associative transfer (i.e., savings from strengthening a common problem node) from mediated transfer (i.e., savings from strengthening a related, mediator problem). Mediation refers to the strategy of solving a problem (e.g., $63 \div 7=$ ?) by reference to a closely related problem (e.g., $7 \times 9=63$ or $63 \div 9=7$ ). Because performance on these occasions depends on the accessibility of the mediator, strengthening of the mediator node produces RT savings (LeFevre \& Morris, 1999; Mauro, LeFevre, \& Morris, 2003).

In response to the evidence that division sometimes is mediated by multiplication, Rickard (2005) advanced a revised IE-r model in which the multiplication IE representation includes a reverse association that provides direct access to factors (e.g., 7 and 5), given presentation of their product (e.g., 35; see also Rusconi, Galfano, Rebonato, \& Umiltà, 2006). Rickard (2005) demonstrated that university students are efficient at factoring (i.e., stating the factors, given the product) and that factoring transfers to the corresponding multiplication fact. Thus, when direct retrieval of a division fact is not possible, division by factoring will be possible if the required reverse association is available. For example, presentation of $56 \div$ 7 would activate the reverse association from 56 to 7 and 8 , with the unique element (8) reported as the quotient (see Campbell, 1999). Given that multiplication retrieval is faster than division retrieval, division by factoring could explain the results of Mauro et al. (2003), who found shorter times to answer large division problems presented in a multiplication format (e.g., $8 \times_{-}=56$ ), as compared with a division format $(56 \div-=8)$.

\section{Identical Elements Model and Simple Addition and Subtraction}

If memory for basic addition and subtraction follows IE principles, the IE predictions are analogous to those for multiplication and division, respectively, with one important unique consideration. Rickard (2005) pointed out that the reverse association would develop for multiplication facts but it would not be likely to develop for addition facts. This asymmetry would occur because, for the single-digit arithmetic facts, most products are associated with a unique pair of factors (e.g., 27 with 3 and 9) or two pairs of factors ( 24 with 3 and 8 or 4 and 6), whereas sums generally do not map onto unique addends. Consequently, practicing multiplication would establish both forward and reverse associations, but practicing addition would not. Without a reverse addition association to mediate subtraction, it follows that addition-based mediational strategies should be relatively infrequent for subtraction.

Experiment 1 here was similar to Experiment 2 in Campbell (1999), in which participants received a large number of transfer tests involving prime-probe problem pairs separated by a number of intervening trials within the same block. The relation between the prime and the probe defined six transfer conditions: addition probe with identical repetition (e.g., prime, $6+9$; probe, $6+9$ ), subtraction probe with identical repetition (prime, $15-6$; probe, $15-6$ ), addition probe with order change (prime, $9+6$; probe, $6+9$ ), subtraction probe with order change (prime, $15-9$; probe, $15-6$ ), addition probe with operation change (prime, $15-9$; probe, $6+9$ ), and subtraction probe with operation change (prime, $6+9$; probe, $15-6)$.

The transfer predictions of the IE model apply specifically to direct memory retrieval, but educated adults report substantial use of procedural strategies for both simple addition (24\%) and subtraction (42\%) (Campbell $\&$ Xue, 2001). In the following experiment, we asked the participants to report their strategy after each trial by selecting from remember, count, transform, or other. Theoretically, selection of the remember category corresponds to direct memory retrieval, whereas selection of one of the other categories corresponds to procedure use (Campbell \& Austin, 2002; Campbell \& Fugelsang, 2001; Campbell, Parker, \& Doetzel, 2004; Campbell \& Xue, 2001). The strategy reports were used to select prime-probe pairs that involved direct retrieval and that, therefore, should present the transfer effects predicted by the IE model.

The predictions for the model were as follows. For addition, RT savings with an order change (e.g., prime, $4+3$; probe, $3+4$ ) should be nearly equal to that observed with identical repetition (prime, $3+4$; probe, $3+4$ ), because commuted addition pairs are composed of identical operands. In contrast, there should be no transfer of savings between the two orders of subtraction problems (prime, $7-3$; probe, $7-4$ ) and no transfer between inverse addition and subtraction problems (prime, $7-3$, and probe, 
$4+3$; or prime, $4+3$, and probe, $7-3$ ). Finally, savings with identical repetition should be greater for subtraction than for addition. It is a virtually universal law of skill acquisition that RT gains per practice trial are greater earlier than later in the learning curve (Logan, 1988; Rickard, 1997). Subtraction is slow relative to addition (Campbell \& Xue, 2001), which implies that for many people, subtraction performance occupies an earlier point on its learning curve, relative to addition. Consequently, identical repetition should produce greater savings for subtraction than for addition.

\section{EXPERIMENT 1}

\section{Method}

\section{Participants}

Forty-eight volunteers (27 women, 21 men) were recruited through the participant pool operated by the Department of Psychology or through advertisements posted around the University of Saskatchewan campus. We described the experiment as a study of simple arithmetic skills. The participants ranged in age from 17 to 54 years $(M=21.9)$, reported normal or corrected-to-normal vision, and were paid $\$ 6$ for their participation.

\section{Apparatus}

The stimuli appeared on two high-resolution monitors controlled by an IBM personal computer, with one monitor viewed by the experimenter and the other by the participant. The stimuli appeared horizontally as white characters against a dark background. The participant sat approximately $50 \mathrm{~cm}$ from the monitor and wore a lapel microphone that activated a relay switch connected to the computer's serial port. The sound-activated relay controlled a software clock accurate to $\pm 1 \mathrm{msec}$.

\section{Stimuli and Design}

The stimuli were addition problems composed of pairs of Arabic digits between 2 and 9 (i.e., $2+2$ through $9+9$ ) and the corresponding subtraction problems $(4-2$ through $18-9)$. The two operands in a problem were separated by the operation sign $(+$ or - ) with adjacent spaces. There are 36 possible pairings of the numbers 2 through 9 when commuted pairs (e.g., $3+8$ and $8+3$ ) are counted as one problem. The set of 36 includes 8 addition tie problems involving a single repeated operand (e.g., $2+2$ and $5+5)$ and 28 addition nontie problems (e.g., $2+3$ or $3+2$ and $5+7$ or $7+$ $5)$. There are 8 corresponding subtraction ties $(4-2$ and $10-5)$ and 28 subtraction nonties $(5-2$ or $5-3$ and $12-7$ or $12-5)$. Digits were $7 \mathrm{~mm}$ high and $3 \mathrm{~mm}$ wide, and the problems were approximately $15 \mathrm{~mm}$ in total width.

The participants received 432 transfer tests involving prime-probe problem pairs separated by a number of intervening trials. The relation between the prime and the probe defined six transfer conditions: addition probe with identical repetition (e.g., prime, $6+9$; probe, $6+9$ ), subtraction probe with identical repetition (prime, $15-6$; probe, $15-6$ ), addition probe with order change (prime, $9+6$; probe, $6+9$ ), subtraction probe with order change (prime, $15-$ 9 ; probe, $15-6$ ), addition probe with operation change (prime, $15-9$; probe, $6+9$ ), and subtraction probe with operation change (prime, $6+9$; probe, $15-6$ ). Tie problems were included in all the conditions to simplify programming but were excluded from analysis because the order change condition does not apply to ties.

The number of trials intervening between the prime and the probe was manipulated, with short prime-probe lags of 4,5 , or 6 intervening trials and long prime-probe lags of 16, 18, and between 12 and 22 trials. The latter, variable lag range corresponded to an aver- age lag of 17 trials. The lag manipulation allowed us to determine whether transfer effects from practicing a single problem were transitory or still measurable after a substantial number of intervening trials spanning about $1-1.5 \mathrm{~min}$.

The participants received 12 blocks of 72 problems. Each block included 36 addition trials and 36 subtraction trials, with 1 primeprobe pair based on each of the 36 number pairs (e.g., $6+9,9+6$, $15-6$, and $15-9$ are based on the 6-9 pair). Across the 12 blocks, each of the 36 number pairs was tested in each of the six transfer conditions, using both a short and a long transfer distance. Within a block, there were 6 prime-probe pairs in each of the six transfer conditions, and three transfer conditions were tested with short and three with long distances. In the following block, the assignment of transfer conditions to short and long lags was reversed. Thus, all 12 transfer-condition-lag combinations were tested six times in each pair of successive odd- and even-numbered blocks. The frequencies of the six transfer conditions in odd- and even-numbered blocks were balanced across participants, as was the assignment of the six transfer conditions to specific short lags (i.e., 4, 5, or 6 trials) and long lags (i.e., 16, 18, and between 12 and 22 trials). Over all the trials, operand order (i.e., $3+6$ vs. $6+3 ; 9-3$ vs. $9-6$ ) was random with respect to transfer condition, but across all the conditions, all the problems were tested exactly six times in each order. Within these constraints, the order of conditions across blocks for each problem and the order of problems in each block were independently randomized for each participant.

\section{Procedure}

Individual participants completed the 70-min experiment with an experimenter present. The instructions described the experiment as a test of speed of simple numerical skills and stated that the task was to state the correct answer as quickly as possible. The participants were advised that occasional errors are normal with speeded responding. The experimenter initiated each trial block, which consisted of a continuous sequence of trials with nothing to differentiate primes and probes. Prior to the first block of trials, the following instructions appeared on the monitor and were read out loud by the experimenter:

After each problem please indicate how you solved the problem by choosing from among the following strategies: Transform, Count, Remember, Other. Say TRANSFORM if you used knowledge of a related problem. Say COUNT if you used a strategy based on counting. Say REMEMBER if the answer seemed to come to you without any intermediate steps, inferences, or calculations. Choose OTHER if you used some other strategy or are uncertain.

For reference during the experiment, the participants also received a sheet of strategy descriptions as follows:

Transform: You solve the problem by referring to a related problem in the same or another operation. For example, you might solve $54 \div 9=$ ? by remembering that $6 \times 9=54$, so $54 \div 9$ must equal 6 . Similar transformation strategies are possible for addition and subtraction.

Count: You solve the problem by counting a certain number of times to get the answer.

Remember: You solve the problem by just remembering or knowing the answer directly from memory without any intervening steps.

Other: You may solve the problem by a strategy unlisted here, or you may be uncertain how you solved the problem.

Prior to each arithmetic trial, a fixation dot appeared and flashed twice over a 1 -sec interval at the center of the screen. The problem appeared (synchronized with the monitor's raster scan) on what would have been the third flash with the operation sign $(+$ or -$)$ at the fixation point. Timing began when the problem appeared and ended when the sound-activated relay was triggered. Triggering the relay caused the problem to disappear immediately. This allowed the experimenter to mark RTs as spoiled because the microphone failed 
to detect the onset of the response. Immediately after the response, the prompt "Strategy Choice" appeared at the center of the screen with the words transform, count, remember, and other centered immediately below. The four words were separated by six spaces and always appeared in the same order. The experimenter recorded the strategy reported by pressing one of four buttons on the computer keyboard. Once the strategy had been recorded and the experimenter had entered the stated arithmetic answer, the screen cleared and displayed the fixation dot for the next trial. No feedback about speed or accuracy was provided during the experiment.

\section{Results and Discussion}

Mean correct RT and percentage of reported use of retrieval each received four-factor lag (short or long) $\times$ operation (subtraction or addition) $\times$ transfer condition (identical repetition, order change, or operation change) $\times$ problem type (prime or probe) ANOVAs. These analyses showed that the lag factor revealed little of theoretical importance; specifically, although performance savings on each dependent measure were generally smaller with the long lag than with the short lag, the patterns of significant and nonsignificant savings were the same for short and long lags, with one exception that we will point out later. Consequently, we will report the results of three-factor operation $\times$ transfer condition $\times$ problem type ANOVAs, with the data collapsed over the lag factor. Subtraction produced more errors overall $(7.5 \%)$ than did addition (4.3\%). Mean error rates were positively correlated with mean RT across conditions $[r(10)=.97]$; therefore, we will not report separate analyses of errors. Reported effects had significance levels of $p<.001$, unless otherwise indicated.

\section{Response Time}

RT savings in each transfer condition were estimated by comparing mean RT on prime trials with the mean RT on yoked probe trials. In the operation change condition, prime-probe pairs involved different operations. Therefore, to estimate RT savings with an operation change, each operation change probe was paired with the corresponding operation change prime from the other operation. To ensure that the prime and probe mean RTs in each operation $\times$ transfer condition cell were based on the same set of specific problems, we excluded both the prime and the probe RTs for a prime-problem pair if either RT was missing, due to an error or voice key failure. On average, 22.9 problems out of a maximum of 28 contributed to each cell.

For each participant, the mean log RT was computed for each cell in the three-factor operation $\times$ transfer condition $\times$ problem type design. We applied the log transformation to reduce effects of outlier RTs. An ANOVA was conducted on the antilogs of the mean log RTs. Applying the antilog transformation restores the normalized means to millisecond units. The mean RTs appear in the top panel of Table 1 . All things being equal, the mean RTs for prime problems should be identical across transfer conditions, but differences arise from the constraint of including the RT for a prime problem only if the RT from the corresponding probe problem was available: With this constraint, transfer conditions that produce probe savings tend to include more difficult prime-probe pairs, because of fewer probe errors. The differences in prime means in Table 1 confirm that mutual exclusion of prime-probe pairs is necessary to avoid inflated estimates of RT savings.

As in previous research (e.g., Campbell \& Xue, 2001), the mean RT for addition (918 msec) was substantially shorter than that for subtraction $(1,154 \mathrm{msec})[F(1,47)=$ 118.92, $\left.M S_{\mathrm{e}}=67,015.96\right]$. The mean RT also was $42 \mathrm{msec}$ shorter on the probe trials $(1,015 \mathrm{msec})$ than on the prime trials $(1,057 \mathrm{msec})$, which confirms overall RT savings [i.e., mean prime RT - mean probe RT; $\left.F(1,47)=131.70, M S_{\mathrm{e}}=2,000.22\right]$. The top panel of Figure 1 shows the mean RT savings and 95\% confidence intervals (Masson \& Loftus, 2003) as a function of operation and transfer condition. As Figure 1 shows, savings varied across transfer conditions, with savings being largest for identical repetition $(80 \mathrm{msec})$, followed by order change (49 $\mathrm{msec})$ and operation change $(-1 \mathrm{msec})$ $\left[F(2,94)=33.70, M S_{\mathrm{e}}=2,411.31\right]$. As was expected, given the greater difficulty of subtraction, as compared with addition, identical repetition produced greater savings for subtraction than for addition.

Table 1

Mean Correct Response Times (in Milliseconds) for All Trials and Retrieval Trials in Experiment 1 by Operation, Transfer Condition, and Problem Type

\begin{tabular}{|c|c|c|c|c|c|c|}
\hline \multirow[b]{2}{*}{$\begin{array}{l}\text { Problem } \\
\text { Type }\end{array}$} & \multicolumn{3}{|c|}{ Subtraction } & \multicolumn{3}{|c|}{ Addition } \\
\hline & $\begin{array}{l}\text { Identical } \\
\text { Repetition }\end{array}$ & $\begin{array}{l}\text { Order } \\
\text { Change }\end{array}$ & $\begin{array}{l}\text { Operation } \\
\text { Change }\end{array}$ & $\begin{array}{l}\text { Identical } \\
\text { Repetition }\end{array}$ & $\begin{array}{c}\text { Order } \\
\text { Change }\end{array}$ & $\begin{array}{c}\text { Operation } \\
\text { Change }\end{array}$ \\
\hline \multicolumn{7}{|c|}{ All Trials $(n=48)$} \\
\hline Prime & 1,192 & 1,188 & 1,151 & 951 & 941 & 922 \\
\hline Probe & 1,085 & 1,144 & 1,162 & 897 & 887 & 913 \\
\hline Savings & $107(13.3)$ & $44(10.4)$ & $-11(10.6)$ & $54(6.5)$ & $55(6.5)$ & $9(8.1)$ \\
\hline \multicolumn{7}{|c|}{ Retrieval Trials $(n=45)$} \\
\hline Prime & 1,004 & 986 & 959 & 856 & 850 & 807 \\
\hline Probe & 923 & 954 & 963 & 817 & 812 & 813 \\
\hline Savings & $81(10.2)$ & $32(8.3)$ & $-4(14.4)$ & $39(6.7)$ & $38(6.5)$ & $-6(8.4)$ \\
\hline
\end{tabular}



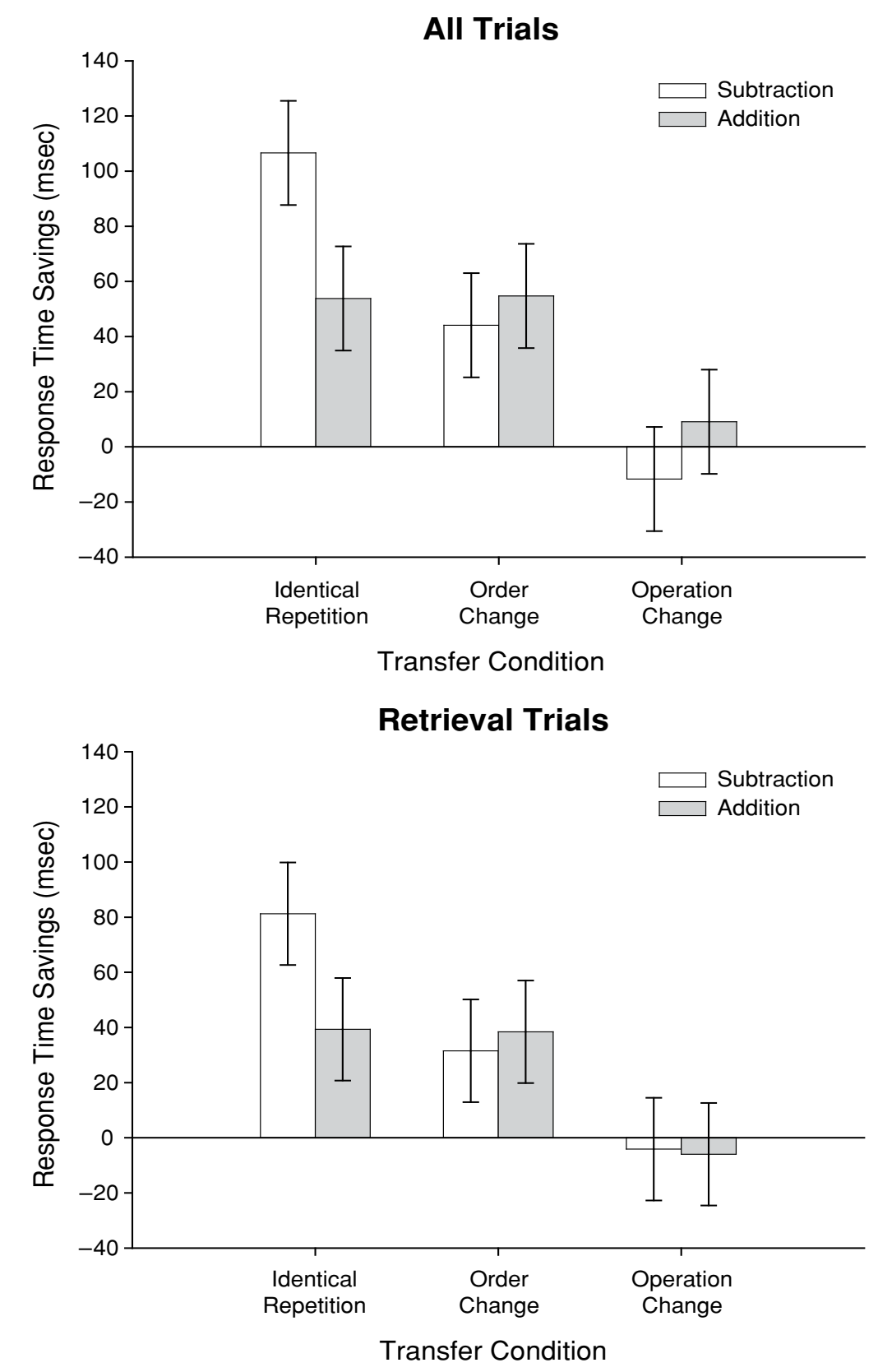

Figure 1. Mean response time savings by operation and transfer condition for all trials and retrieval trials in Experiment 1. Error bars are $95 \%$ confidence intervals (Masson \& Loftus, 2003).

Moreover, the operation $\times$ transfer condition $\times$ problem type interaction predicted by the IE model was significant $\left[F(2,94)=9.92, M S_{\mathrm{e}}=1,924.23\right]$. Specifically, for addition, identical repetition and order change produced equivalent savings, and there were no RT savings for addition with an operation change. In contrast, for subtraction, identical repetition produced much greater savings than did order change. As with addition, there were no subtraction RT savings with an operation change.
RT savings on retrieval trials. The IE model correctly predicted the operation $\times$ transfer condition $\times$ problem type interaction, but the significant $44-\mathrm{msec}$ savings effect for subtraction probes with an order change (see Figure 1) is not predicted by the model. These savings might be attributable, however, to procedural strategies that are not relevant to the model's transfer predictions for retrievalbased performance. Consequently, we redid the RT analysis, including only trials on which both the prime and the 
probe problems were reportedly solved by direct retrieval. We will report a detailed analysis of strategy reports later on; for now, it is sufficient to indicate that, on average, direct retrieval was reported for $64 \%$ of the subtraction trials and $79 \%$ of the addition trials.

The means for the retrieval-only RT analysis appear in the bottom panel of Table 1. Exclusion of nonretrieval prime-probe pairs produced empty cells for 3 participants. Consequently, the retrieval-only RT means in Table 1 and the corresponding ANOVA were based on an $n$ of 45 . The overall mean RT for retrieval trials was $896 \mathrm{msec}$, substantially shorter than the overall 1,036-msec mean for the all-trials analysis. This would be expected because direct memory retrieval generally is faster than use of a procedural strategy for simple arithmetic and retrieval is more likely for the numerically smaller, better memorized number facts (Campbell \& Xue, 2001; LeFevre, Sadesky, \& Bisanz, 1996).

Mean RT savings and 95\% confidence intervals for the retrieval-only RT analysis appear in the bottom panel of Figure 1 . When the analysis included only retrieval trials, the pattern of savings was similar to the all-trials analysis, except that savings generally were smaller with procedure trials excluded. Indeed, the pattern of significant RT savings (see Figure 1) was the same as that in the all-trials analysis, including the three-way interaction of operation, transfer condition, and problem predicted by the IE model $\left[F(2,88)=3.14, M S_{\mathrm{e}}=2,425.68, p=.048\right]$. Specifically, for addition, there were equivalent RT savings with identical repetition and order change but no savings with an operation change. For subtraction, savings with identical repetition were greater than those with an order change and, as with addition, there were no RT savings with an operation change. Nonetheless, there remained evidence of RT savings for subtraction probes with an order change (see Figure 1). This effect occurred, however, only with short prime-probe lags of 4-6 trials (56-msec savings), but not with long lags of 17 trials on average (8-msec savings). For all the other cells depicted in Figure 1, the pattern of significant or nonsignificant savings (based on $95 \%$ confidence intervals) was the same for the short and the long lag intervals, although savings were consistently about $50 \%$ smaller with the long lags, relative to the short lags.

Discussion of RT savings. The pattern of results closely resembled the pattern predicted by the IE model. Transfer with identical or reversed addition pairs was equivalent. There was no transfer between addition and subtraction in either direction, despite the fact that the relatively short prime-probe lags used would make the experiment sensitive to both associative and mediated transfer. Finally, subtraction with identical repetition produced much greater savings than did subtraction with an order change, as was expected.

Nonetheless, the retrieval-only analysis reduced, but did not eliminate, subtraction savings with an order change. This finding implies that retrieval of one order of a subtraction fact (e.g., $15-8=7$ ) facilitated retrieval for the other order $(15-7=8)$ over shorter lags. Although potentially anomalous for the IE model, we do not think at this time that this result should be counted as a critical exception for the model. The strategy report method we used generally can distinguish direct retrieval from use of procedures (see Campbell \& Austin, 2002; Campbell et al., 2004; Campbell \& Xue, 2001), but it remains possible that some procedure strategies were classified as retrieval, which would allow mediated transfer effects to contaminate the retrieval-only analysis. This explanation is speculative, but given that the magnitude of subtraction savings with an order change was about one third of that with identical repetition, the short-lag RT transfer for subtraction probes with an order change probably should not be counted as a critical violation of IE model predictions. Indeed, the much greater subtraction RT savings with identical repetition than with an order change implies that savings with identical repetition involved a more direct or consistent transfer mechanism (i.e., strengthening of a common memory node) than did subtraction savings with an order change.

In general, the pattern of RT savings was consistent with the IE model; nonetheless, the results indicate that the participants could efficiently exploit the relationship between inverse subtraction problems to facilitate performance. There was no evidence in the RT analysis, however, that people similarly exploited their knowledge of inverse addition and subtraction pairs. To pursue these issues we conducted a detailed analysis of the strategy reports.

\section{Strategy Reports}

The participants selected the remember strategy most often $(72.0 \%$ of the trials), followed by count $(16.9 \%)$, transform (10.1\%), and other (1.0\%). Table 2 presents the mean percentages of selection of retrieval (i.e., the remember strategy item), transform, and count as a function of operation, transfer condition, and problem type (prime or probe). Our participants reported direct memory retrieval much more for addition $(79.3 \%)$ than for subtraction $(63.8 \%)$. This mainly reflected more reported use of transformation for subtraction $(16.0 \%)$ than for addition $(4.2 \%)$, whereas reported use of counting was about the same for subtraction (18.6\%) and addition (15.2\%). For simple addition, the $79 \%$ direct memory retrieval was slightly higher than in previous research in which young North American adults had been tested (e.g., $76 \%$ for non-Asian Canadians in Campbell \& Xue, 2001; 69\% in Campbell \& Timm, 2000; 73\% in Geary, 1996; 66\% in Hecht, 1999; 71\% in LeFevre, Sadesky, \& Bisanz, 1996). The $64 \%$ retrieval reported for subtraction was similar to that in Campbell and Xue for non-Asian Canadians (57\%) and to the $71 \%$ reported by Geary, Frensch, and Wiley (1993) for American university students but was considerably lower than the $85 \%$ retrieval for adults' subtraction observed by Seyler, Kirk, and Ashcraft (2003).

There also were transfer effects on reported retrieval. Figure 2 presents mean retrieval savings (i.e., probe retrieval percentage - prime retrieval percentage) and 95\% 
Table 2

Mean Percentages of Retrieval, Transform, and Count Strategies Reported in Experiment 1 by Operation, Transfer Condition, and Problem Type

\begin{tabular}{|c|c|c|c|c|c|c|}
\hline \multirow[b]{2}{*}{$\begin{array}{l}\text { Problem } \\
\text { Type }\end{array}$} & \multicolumn{3}{|c|}{ Subtraction } & \multicolumn{3}{|c|}{ Addition } \\
\hline & $\begin{array}{l}\text { Identical } \\
\text { Repetition }\end{array}$ & $\begin{array}{c}\text { Order } \\
\text { Change }\end{array}$ & $\begin{array}{c}\text { Operation } \\
\text { Change }\end{array}$ & $\begin{array}{l}\text { Identical } \\
\text { Repetition }\end{array}$ & $\begin{array}{c}\text { Order } \\
\text { Change }\end{array}$ & $\begin{array}{c}\text { Operation } \\
\text { Change }\end{array}$ \\
\hline \multicolumn{7}{|c|}{ Retrieval } \\
\hline Prime & 62.1 & 62.0 & 61.2 & 78.4 & 77.9 & 79.4 \\
\hline Probe & 68.5 & 65.6 & 63.5 & 81.5 & 80.3 & 78.5 \\
\hline Difference & $6.4(1.0)$ & $3.6(0.9)$ & $2.3(1.1)$ & $3.1(0.6)$ & $2.4(0.7)$ & $-0.9(0.8)$ \\
\hline \multicolumn{7}{|c|}{ Transform } \\
\hline Prime & 16.5 & 17.1 & 17.0 & 4.4 & 4.1 & 4.2 \\
\hline Probe & 14.3 & 15.7 & 15.3 & 3.7 & 4.2 & 4.7 \\
\hline Difference & $-2.2(0.8)$ & $-1.4(0.7)$ & $-1.7(0.9)$ & $-0.7(0.3)$ & $0.2(0.3)$ & $0.5(0.4)$ \\
\hline \multicolumn{7}{|c|}{ Count } \\
\hline Prime & 19.4 & 19.4 & 20.0 & 16.0 & 16.6 & 15.4 \\
\hline Probe & 15.5 & 17.1 & 19.9 & 13.6 & 13.9 & 15.4 \\
\hline Difference & $-3.9(0.8)$ & $-2.3(0.7)$ & $-0.1(0.9)$ & $-2.4(0.6)$ & $-2.7(0.6)$ & $0.0(0.8)$ \\
\hline
\end{tabular}

Note-Difference $=$ probe - prime. Standard errors of mean differences are shown in parentheses.

confidence intervals as a function of operation and transfer condition. ${ }^{1}$ An operation $\times$ transfer $\times$ problem type ANOVA of percentages of selection of remember indicated that, overall, the participants reported retrieval more on probe $(73.0 \%)$ than on prime $(70.2 \%)$ trials $[F(1,47)=$ $\left.53.52, M S_{\mathrm{e}}=21.42\right]$, and this corresponded to small decreases in both transformation $(-0.9 \%)$ and counting $(-1.9 \%)$ (see Table 2). Retrieval savings were largest for identical repetition $(4.7 \%)$, followed by order change $(3.0 \%)$ and operation change $(1.6 \%)[F(2,94)=10.32$, $\left.M S_{\mathrm{e}}=19.08\right]$. The effect of primes on probe retrieval was greater for subtraction $(5.1 \%)$ than for addition $(1.5 \%)$ $\left[F(2,94)=11.93, M S_{\mathrm{e}}=20.27, p=.001\right]$, a difference attributable, in part, to the fact that use of retrieval for addition was much closer to the $100 \%$ ceiling. As Figure 2 shows, both subtraction and addition probes showed significant retrieval savings with both identical repetition and order change. Subtraction probes, but not addition probes, however, showed retrieval savings with an operation change. An operation $\times$ problem type analysis of the operation change condition confirmed a significant operation $\times$ problem type interaction $[F(1,47)=4.98$, $\left.M S_{\mathrm{e}}=24.55, p=.03\right]$, which corresponds to retrieval savings with an operation change for subtraction, but not for addition.

Discussion of retrieval savings. As is shown in Figure 2, the analysis of retrieval savings confirmed several predictions of the IE model. For addition, retrieval savings were equivalent for identical repetition and order change, and there were no retrieval savings with an operation change. For subtraction, retrieval savings were greater with identical repetition, relative to both order change and operation change, and the latter conditions did not differ. Nonetheless, as in the RT analysis, there were subtraction savings not predicted by the IE model. Specifically, the analysis demonstrated retrieval savings for subtraction probes following both an order change and an operation change. These effects were small, however, as compared with subtraction retrieval savings with identical repetition. Moreover, subtraction retrieval savings with an order change and operation change are not inherently at odds with the IE model, because retrieval savings could arise from procedure use (i.e., prime solved by procedure and

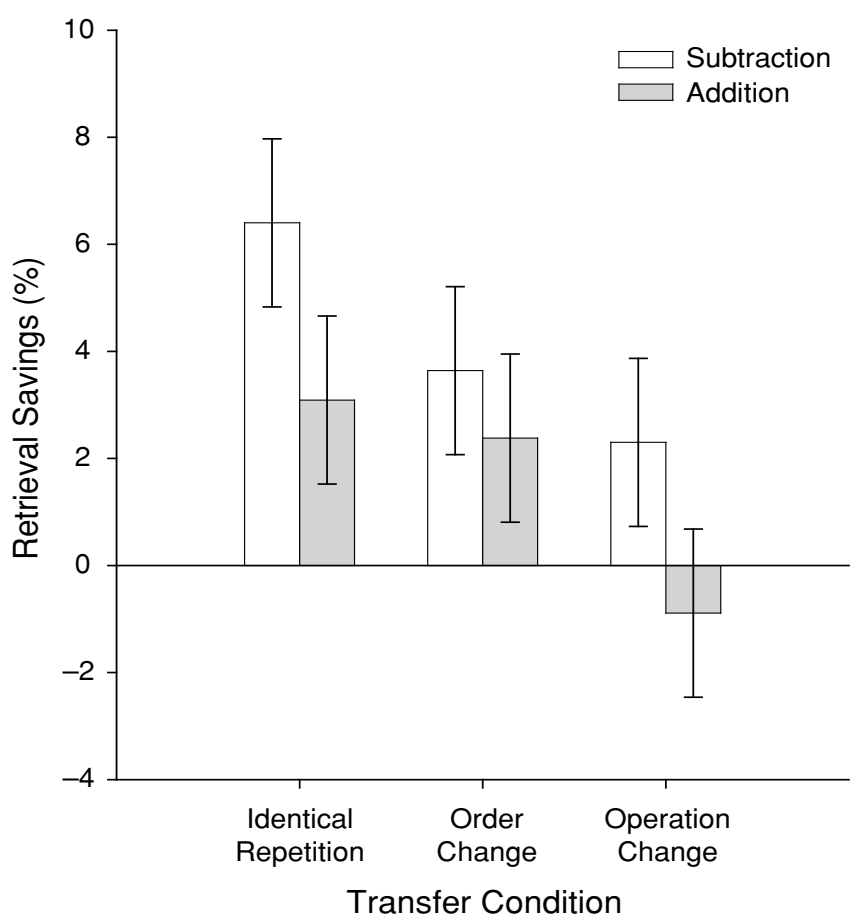

Figure 2. Mean retrieval savings by operation and transfer condition in Experiment 1. Error bars are $95 \%$ confidence intervals (Masson \& Loftus, 2003). 
probe solved by retrieval). Thus, the pattern of savings in Figure 2 is compatible with an IE model for addition and subtraction.

\section{EXPERIMENT 2}

Experiment 1 largely confirmed the RT predictions of the IE model as applied to simple addition and subtraction. Experiment 2 revisited the multiplication and division transfer experiment reported by Campbell (1999) in order to pursue observed RT savings in the multiplicationto-division transfer condition (e.g., prime, $7 \times 9$; probe, $63 \div 7$ ). The following experiment was similar to that in Campbell (1999) but included only identical repetition and operation change transfer conditions. The critical difference was that here, we asked the participants to indicate whether they solved each problem by direct retrieval or by a procedural strategy. As in Experiment 1, the strategy reports were used to select prime-probe pairs that involved direct retrieval and that should present only the transfer effects predicted by the IE model. Whereas we might observe mediated transfer of RT savings between multiplication and division when all the trials are analyzed, according to the IE model, there should be no cross-operation transfer when the analysis includes only problems solved by direct retrieval.

\section{Method}

\section{Participants}

Fifty-six volunteers (38 women, 18 men) were recruited from the University of Saskatchewan Psychology Department participant pool and received course credit for participation in the experiment. The participant sign-up sheet described the experiment as a test of speed for simple arithmetic. The participants' mean age was 19.5 years.

\section{Apparatus and Stimuli}

The apparatus was the same as that in Experiment 1. The stimuli were multiplication problems composed of pairs of digits between 2 and 9 and the corresponding division problems. There are 28 possible combinations of the numbers $2-9$ (excluding ties, e.g., $2 \times$ $2,3 \times 3$, etc.). The computer program that created the sequences of interleaved prime-probe pairs was designed to handle 24 pairs for counterbalancing purposes. Consequently, the $2-3,2-4,2-5$, and 2-6 pairs were excluded. We excluded small problems because the cross-operation transfer between multiplication and division observed in previous research was more pronounced for large problems (Campbell, 1999; LeFevre \& Morris, 1999). The stimuli were based on the 24 remaining pairs. Each pair was the basis for two multiplication and two division problems (e.g., the 2-7 pair gives $2 \times 7,7 \times 2,14 \div 2$, and $14 \div 7$ ). The problems were presented horizontally, with the two operands separated by the operation sign, with adjacent blank spaces (e.g., $6 \times 8,48 \div 6$ ). Digits were $7 \mathrm{~mm}$ high $\times 3 \mathrm{~mm}$ wide.

\section{Design}

There were eight blocks of 48 problems, for a total of 384 trials. Each block consisted of 24 paired prime and probe problems separated by a number of intervening trials. The prime-probe lags used were $4,6,8$, or 9-12 trials. The latter, variable lag condition facilitated creation of randomized trial sequences in which 24 prime-probe pairs were interleaved into a continuous series of tri- als, with nothing to distinguish the primes from the probes. Each prime-probe pair represented one of four transfer conditions: multiplication probe with identical repetition (e.g., prime, $7 \times 8$; probe, $7 \times 8$ ), division probe with identical repetition (prime, $56 \div 8$; probe, $56 \div 8$ ), multiplication probe with operation change (prime, $56 \div 8$; probe, $7 \times 8$ ), and division probe with operation change (prime, $7 \times 8$; probe, $56 \div 7$ ). Since our focus for Experiment 2 was cross-operation transfer, we did not include within-operation order change conditions.

The following design was implemented in the first four blocks and was replicated over the second group of four blocks: Within each block, each of the 24 operand pairs was tested once in one of the four transfer conditions, and there were 12 prime-probe pairs at each of the four lags. Across the four blocks, each transfer condition was tested six times at each of the four lags, and all 24 operand pairs (i.e., problems) were tested in each transfer condition. The 24 operand pairs were tested once at each of the four specific lags across all conditions. For each transfer condition, half the multiplication problems had the smaller number on the left (e.g., $3 \times 8)$, and half of the division problems involved the min-divisor order $(24 \div 3)$. In all, each multiplication and division problem was tested twice in each operand order. Within these constraints, operand order, the order in which problems appeared in each block, and the assignment of operand pairs to transfer conditions across blocks were independently randomized for each participant.

\section{Procedure}

The participants were individually tested by an experimenter who remained present throughout the procedure, which required about $1 \mathrm{~h}$. Initial instructions explained that the participants would receive several hundred simple multiplication and division problems and that after each problem, they would indicate whether they had solved the problem by remembering the answer or had used a strategy to derive the answer. The participants named 20 random numbers from 1 through 40, which appeared on the screen. This number-naming task allowed the experimenter to adjust the sensitivity of the soundactivated timer. After the naming task, the following instructions appeared on the screen:

There will be 8 blocks of 48 simple arithmetic problems. A random half of the trials are division and the other half are multiplication. Try to state the correct answer accurately but quickly. A warning dot will flash before each problem appears. Occasional errors are normal and should not concern you. After each problem please indicate whether you used a STRATEGY or simply REMEMBERED the answer. Say STRATEGY if any intermediate step, inference, or calculation was used to obtain the answer. Say REMEMBERED if the answer seemed to come to you without any intermediate step, inferences or calculations.

The experimenter initiated each block of trials. A warning dot flashed twice over a 1-sec interval at the center of the screen for each trial. The problem then appeared with the operation sign $(\div$ or $\times)$ at the fixation point. Response timing began with problem onset and terminated with the participant's response. The problem was then replaced with the strategy categories presented on a single horizontal line in the following order: Strategy? Uncertain? or Remembered? The next trial began after the experimenter had pressed a key to record the strategy selection and entered the participant's arithmetic answer. The participant was offered a brief rest between blocks. There was no feedback about performance.

\section{Results and Discussion}

The data were submitted to three-way repeated measures ANOVAs with factors of operation (multiplication or division), transfer condition (operation change or identical repetition), and problem type (prime or probe). Error rates $(6.1 \%$ for division, $5.5 \%$ for multiplication) were 
Table 3

Mean Correct Response Times (RTs, in Milliseconds) for All Trials and Retrieval Trials, and Percentages of Retrievals Reported in Experiment 2 by Operation, Transfer Condition, and Problem Type

\begin{tabular}{lcccc}
\hline & \multicolumn{2}{c}{ Division } & \multicolumn{2}{c}{ Multiplication } \\
\cline { 2 - 3 } $\begin{array}{c}\text { Problem } \\
\text { Type }\end{array}$ & $\begin{array}{c}\text { Identical } \\
\text { Repetition }\end{array}$ & $\begin{array}{c}\text { Operation } \\
\text { Change }\end{array}$ & $\begin{array}{c}\text { Identical } \\
\text { Repetition }\end{array}$ & $\begin{array}{c}\text { Operation } \\
\text { Change }\end{array}$ \\
\hline \multicolumn{5}{c}{ RTs on All Trials } \\
Prime & 1,410 & 1,363 & 1,225 & 1,174 \\
Probe & 1,238 & 1,322 & 1,107 & 1,133 \\
$\quad$ Savings & $172(15.2)$ & $41(10.5)$ & $118(9.4)$ & $41(12.7)$ \\
\multicolumn{5}{c}{ RTs on Retrieval Trials } \\
Prime & 1,254 & 1,200 & 1,119 & 1,057 \\
Probe & 1,127 & 1,193 & 1,031 & 1,037 \\
Savings & $127(15.0)$ & $7(15.0)$ & $88(8.7)$ & $20(13.4)$ \\
\multicolumn{5}{c}{ Percentages of Retrievals Reported } \\
Prime & 76.7 & 77.3 & 82.0 & 82.9 \\
Probe & 83.2 & 80.1 & 88.4 & 87.2 \\
Savings & $6.5(1.2)$ & $2.8(1.1)$ & $6.4(0.8)$ & $4.3(0.9)$ \\
\hline
\end{tabular}

Note-Savings $=$ prime - probe for RT, probe - prime for percentage of retrieval. Standard errors of mean savings are shown in parentheses.

positively correlated with mean RT across conditions $[r(6)=.67]$. Therefore, we focused on RTs for correct trials and percentages of retrieval reported (i.e., selection of remember).

\section{Response Time}

The RT data were processed as in Experiment 1. A mean of 19.6 problems out of a maximum of 24 contributed to each cell. The RT means for each operation $\times$ transfer condition $\times$ problem type cell appear in the top of Table 3 . As in previous research (e.g., Campbell \& Xue, 2001), mean RT for multiplication $(1,160 \mathrm{msec})$ was shorter than that for division $(1,333 \mathrm{msec})\left[F(1,55)=54.68, M S_{\mathrm{e}}=\right.$ $61,360.78]$. Responses on probe trials $(1,200 \mathrm{msec})$ were faster than those on prime trials $(1,293 \mathrm{msec})$, confirming overall RT savings $\left[F(1,55)=209.97, M S_{\mathrm{e}}=4,606.27\right]$. The top panel of Figure 3 shows the mean RT savings and $95 \%$ confidence intervals as a function of operation and transfer condition.

As Figure 3 shows, the patterns of RT savings for multiplication and division were as predicted by the IE model, inasmuch as savings with identical repetition were greater than those with an operation change $[F(1,55)=6.25$, $\left.M S_{\mathrm{e}}=3,164.89, p=.015\right]$. The operation $\times$ transfer $\times$ problem type interaction was also significant $[F(1,55)=$ $\left.5.18, M S_{\mathrm{e}}=7,884.66, p=.027\right]$. The three-way effect occurred because savings with identical repetition were greater for division (172 $\mathrm{msec}$ ) than for multiplication (118 $\mathrm{msec}$ ), whereas RT savings with operation change were the same for division (41 msec) and multiplication (41 msec). Division performance occupies an earlier point on its learning curve, relative to multiplication; consequently, according to theories of practice-related speedup (e.g., Logan, 1988; Rickard, 1997), identical repetition would produce greater savings for division than for multiplication.
Using a similar paradigm, Campbell (1999) found multiplication-to-division transfer only for large problems. To pursue this, we examined the operation change conditions separately for small (product or dividend $\leq 25$ ) and large (product or dividend $>25$ ) problems. For large problems, there were substantial savings both for multiplicationto-division transfer [83 msec; $t(55)=4.02, S E=20.6, p<$ $.001]$ and division-to-multiplication transfer [64 $\mathrm{msec}$; $t(55)=2.77, S E=23.0, p=.008]$ that were not statistically different $[t(55)=0.61, S E=31.5, p>.5]$. For small problems, however, there was no evidence of savings for either multiplication-to-division transfer $[-0.1 \mathrm{msec}$; $t(55)=-0.06, S E=14.6]$ or division-to-multiplication transfer [19.9 msec; $t(55)=1.48, S E=13.5, p=.15]$. The operation change RT savings for large multiplication and division problems demonstrate that recently solving the inverse problem from the other operation facilitated performance.

RT savings on retrieval trials. As in Experiment 1, we redid the RT analysis but included only cases in which both the prime and the probe problems were reportedly solved by direct retrieval. On average, direct retrieval was reported for $79 \%$ of the division trials and $85 \%$ of the multiplication trials. The means for the retrieval-only RT analysis appear in the center part of Table 3. With procedure trials excluded, overall mean RT decreased by $120 \mathrm{msec}$ (1,247 msec for all trials vs. 1,127 msec for retrieval only). As in Experiment 1, this decrease reflects the fact that use of retrieval generally is faster than use of procedural strategies and more likely to involve numerically smaller, relatively easy problems (Campbell \& Xue, 2001).

The mean RT savings and 95\% confidence intervals for the retrieval-only RT analysis are presented in the lower panel of Figure 3 . The analysis confirmed the three-way operation $\times$ transfer condition $\times$ problem type interaction $\left[F(1,55)=5.84, M S_{\mathrm{e}}=6,384.95, p=.019\right]$ : Savings with identical repetition were greater for division $(127 \mathrm{msec})$ than for multiplication $(88 \mathrm{msec})$, whereas nominal savings with operation change were the same for division (7 $\mathrm{msec}$ ) and multiplication (21 msec). As Figure 4 shows, however, neither division nor multiplication evidenced significant savings with an operation change once procedure trials were excluded from the analysis.

Discussion of RT savings. With procedure trials excluded, there remained large RT savings for both multiplication and division with identical repetition, but no overall RT savings for either operation with an operation change. In contrast, in the all-trials analysis with procedures included, there were significant RT savings in the operation change condition for both multiplication and division. Therefore, the cross-operation transfer was associated primarily with procedural strategies, rather than with direct retrieval. As in LeFevre and Morris's (1999) study, Experiment 2 indicated transfer in both directions between multiplication and division, especially for larger problems. Campbell (1999) found clear evidence only of multiplication-to-division transfer, but given the results of Experiment 2, and LeFevre and Morris's results, the 

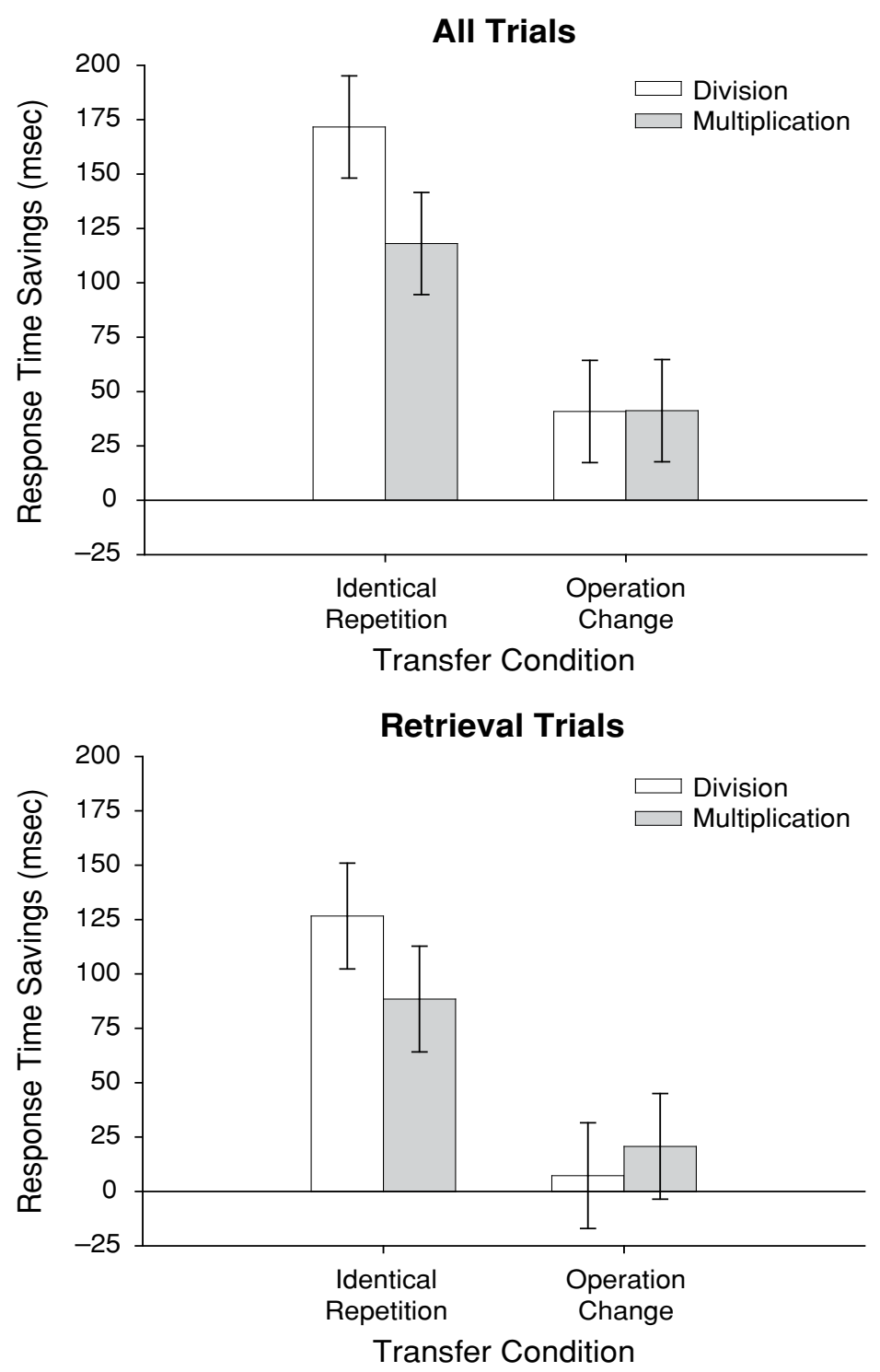

Figure 3. Mean response time savings by operation and transfer condition for all trials and retrieval trials in Experiment 2. Error bars are $\mathbf{9 5 \%}$ confidence intervals (Masson \& Loftus, 2003).

weight of evidence supports transfer between multiplication and division in both directions.

\section{Strategy Reports}

If the participants sometimes used multiplication to mediate division, we would expect division-to-multiplication retrieval savings, because such mediation implies that the multiplication probe problem was processed during the preceding division prime trial. This would increase the probability of direct retrieval of the multiplication probe. Similarly, we would expect multiplication-to-division retrieval savings if processing of the multiplication prime involved strengthening of the corresponding division probe, although this seems less likely a priori. In addition, we would expect greater retrieval savings with identical repetition than with an order change, if identical repetition provides direct associative transfer whereas savings with an order change reflects less direct, mediated transfer.

The participants selected remembered most often ( $82.2 \%$ of trials), followed by strategy $(15.6 \%)$ and uncertain $(2.2 \%)$. The bottom part of Table 3 presents the mean percentages of selection of remembered (i.e., direct retrieval) as a function of operation, transfer condition, and problem type (prime or probe). The corresponding ANOVA confirmed more reported retrieval for multiplication $(85.1 \%)$ than for division $(79.3 \%)[F(1,55)=13.91$, $\left.M S_{\mathrm{e}}=270.12\right]$. These rates of retrieval for multiplication and division are not unusual, although the reported rates in previous research have been quite variable: for multiplication by North American university students, $96 \%$ for 


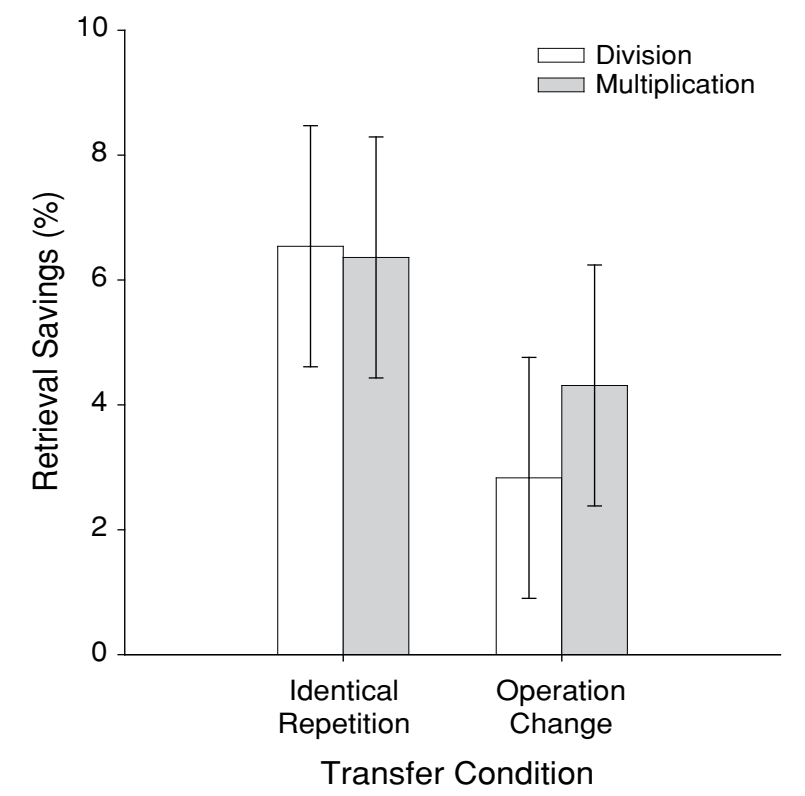

Figure 4. Mean retrieval savings by operation and transfer condition in Experiment 2. Error bars are $95 \%$ confidence intervals (Masson \& Loftus, 2003).

non-Asian Canadians in Campbell and Xue (2001); 87\% in Campbell and Timm (2000); 78\% in Hecht (1999); 85\% in LeFevre, Bisanz, et al. (1996); and 59\% in LeFevre and Morris (1999). For simple division, LeFevre and Morris found $55 \%$ reported retrieval for Canadian undergraduates, and Campbell and Xue found 57\% for non-Asian Canadians, both of which are considerably lower than the $79 \%$ observed here. Nonetheless the retrieval rate for division in the present experiment was not as high as the $90 \%$ retrieval for division reported by Campbell and Timm.

The participants reported retrieval more for probes $(84.7 \%)$ than for primes $(79.7 \%)$, which confirms retrieval savings $\left[F(1,55)=83.85, M S_{\mathrm{e}}=33.54\right]$. Figure 4 presents the mean retrieval savings (i.e., probe retrieval percentage - prime retrieval percentage) and $95 \%$ confidence intervals as a function of operation and transfer condition. Retrieval savings were greater with identical repetition $(6.5 \%)$ than with operation change $(3.6 \%)$ $\left[F(1,55)=6.75, M S_{\mathrm{e}}=34.38, p=.012\right]$. Nonetheless, as Figure 4 shows, there were significant retrieval savings with both identical repetition and operation change. There was no evidence of a three-way interaction of operation, transfer condition, and problem type $[F(1,55)=0.90$, $\left.M S_{\mathrm{e}}=43.79\right]$. As in the RT analysis, we redid the retrieval savings analysis, including only numerically larger, more difficult problems (product or dividend $>25$ ). The pattern of statistically significant effects was exactly the same as in Figure 4, although the mean savings were somewhat larger for the larger problems. This would be expected, since larger problems are most likely to be solved by procedures (Campbell \& Xue, 2001; LeFevre \& Morris, $1999)$ and, therefore, provide more opportunities to display retrieval savings.
Discussion of retrieval savings. As was anticipated, identical repetition produced greater retrieval savings than did operation change. This would be expected if retrieval savings with identical repetition involved a more direct transfer mechanism (i.e., strengthening of a common memory node) than did retrieval savings with an order change. Nonetheless, both operations presented retrieval savings with an operation change. Division-to-multiplication retrieval savings were expected, given the RT evidence that multiplication sometimes mediated division. The reverse effectmultiplication-to-division retrieval savings - would occur if division sometimes mediated multiplication, although we would not predict this, because knowledge and performance are better for multiplication than for division. Alternatively, given the experimental prevalence of inverse division and multiplication problems appearing within a few trials of one another, it would not be surprising if the participants occasionally thought about the inverse division problem after a multiplication trial. Such incidental rehearsal might be sufficient to produce the small multiplication-todivision retrieval savings observed.

\section{GENERAL DISCUSSION}

The General Discussion is divided into three sections. We first will consider the implications of Experiment 1 for extending the IE model to include addition and subtraction. Second, we will discuss the implications of Experiment 2 for the IE model of multiplication and division facts and will explain how a revised IE-r model (Rickard, 2005) can account for the complex pattern of facilitative transfer effects observed across all four arithmetic operations. Finally, we will consider directions for future development of the IE-r model.

\section{Identical Elements Model for Addition and Subtraction}

Experiment 1 confirmed predictions of the IE model as applied to simple addition and subtraction: There were equivalent RT savings for addition with identical repetition and addition with an order change, greater savings for subtraction with identical repetition than for subtraction with an order change, and no transfer of savings between addition and subtraction. That there should be equivalent savings for addition with identical repetition or with an order change is consistent with the assumption that only one order is represented in memory (Butterworth, Zorzi, Girelli, \& Jonkheere, 2001). The only potentially problematic result for the IE model was evidence in the RT analysis of retrieval trials that practicing one order of a subtraction problem (e.g., $15-8=7$ ) facilitated performance of the other $(15-7=8)$. Nonetheless, the subtraction savings with an order change were much smaller than the subtraction savings with identical repetition and were observed only over short lags of four to six trials, whereas all other RT transfer effects were present at both the short and the longer lags. The small short-lag RT savings for subtraction with an order change are plausibly 
attributable to mediated transfer, rather than to associative transfer, and this represents, at most, a weak challenge to the IE model.

The analysis of retrieval savings in Experiment 1 also confirmed predictions of the IE model. For addition, retrieval savings were equivalent for identical repetition and order change, and there were no addition retrieval savings with an operation change. For subtraction, retrieval savings were greater with identical repetition, relative to both order change and operation change, and the latter did not differ. The analysis also demonstrated retrieval savings for subtraction probes following both an order change and an operation change. These effects are not predicted by the IE model but are compatible with the model, because either associative or mediated transfer could contribute to retrieval savings.

Savings for subtraction probes with an order change appeared both in the retrieval savings analysis and in the RT analysis. Increased use of retrieval would be expected to produce shorter RTs, because retrieval is generally faster than use of procedures. Savings on subtraction probes with an operation change, however, appeared only in retrieval savings and not in RT savings. The retrieval savings were small, however: $2.3 \%$, which is an average increase in reported retrieval of only 0.6 problems. Consequently, the increase in retrieval usage might have been too small to be expressed in RT. Retrieval savings apparently can provide a more sensitive (i.e., direct) measure than can RT of weak transfer effects on retrieval usage. Whereas there were addition-to-subtraction retrieval savings, there was no evidence that solving a subtraction problem increased the probability of retrieval for the inverse addition problem. This asymmetry might reflect the fact that the cognitive events that mediated cross-operation retrieval savings were quite rare (e.g., incidental rehearsal of the inverse problem), and since retrieval for addition was much closer to ceiling than was subtraction, addition performance provided fewer opportunities for retrieval savings.

In summary, the results of Experiment 1 provided good support for an extension of the IE model to addition and subtraction. Despite evidence that multiplication can involve different neural mechanisms than do addition and subtraction (e.g., Cohen, Dehaene, Chochon, Lehericy, \& Naccache, 2000; van Harskamp \& Cipolotti, 2001), the present results imply that, when performance is based on direct memory retrieval, the IE principles apply to all four arithmetic operations. Furthermore, the transfer pattern predicted by the IE model was observed without massive amounts of experimental practice (see Rickard \& Bourne, 1996). Thus, the IE model quite accurately characterized our participants' preexperimental addition and subtraction memory.

\section{Identical Elements Model for Multiplication and Division}

The results of Experiment 2 provided additional evidence for the IE model's application to multiplication and division. Specifically, in Experiment 2, the source of the facilitative transfer between multiplication and division found in previous research was investigated. Campbell (1999) used a transfer paradigm similar to that in Experiment 2 and found RT savings in the multiplication-todivision transfer condition for large problems (e.g., prime, $7 \times 9$; probe, $63 \div 7$ ). LeFevre and Morris (1999) found that practicing a block of division or a block of multiplication problems produced RT savings for the inverse problems in the other operation, especially for large problems. These cross-operation transfer effects in multiplication and division potentially raise problems for the IE model if they reflect transfer that is due to direct retrieval, rather than transfer that is due to mediational strategies.

In Experiment 2, the all-trials analysis of RT savings demonstrated interoperation savings, both from multiplication to division and from division to multiplication. As in previous research (Campbell, 1999; LeFevre \& Morris, 1999), these effects were associated with large division problems. These interoperation RT savings, however, depended on inclusion of problems that reportedly had been solved by procedural strategies. When the analysis included only cases in which prime-probe pairs reportedly had been solved by retrieval, there were no significant interoperation RT savings in either direction. Thus, the transfer effects were as predicted by the IE model when transfer was measured for retrieval trials.

Rickard's (2005) revised IE model (IE-r) also explains the finding here of interoperation transfer in both directions for multiplication and division and the absence of such transfer for addition and subtraction. This follows under the IE-r assumption that along with forward associations linking factors to products, multiplication representations also incorporate a reverse association linking products to factors (see also Rusconi et al., 2006). The IE-r model assumes that practicing either multiplication or factoring strengthens both the forward and the reverse associations, although strengthening is greater for the direction practiced. This implies that strengthening the forward association for a multiplication fact (e.g., practicing $8 \times 7=56$ ) will produce savings for the inverse division problem $(56 \div 7)$ if answering it is based on division by factoring. Conversely, division by factoring will strengthen the forward association for the corresponding multiplication fact and will produce transfer from division to multiplication. Thus, the IE-r model provides a mechanism for the interoperation transfer in both directions observed here in Experiment 2 and by LeFevre and Morris (1999). In contrast, Rickard (2005) argued that addition fact representations would not include a reverse association from sum to addends; consequently, unlike division and multiplication, there is not a convenient mechanism for solving subtraction problems, given the inverse addition fact. Thus, the revised IE-r model explains why we observed no interoperation transfer of savings for addition and subtraction (Experiment 1) but observed interoperation transfer in both directions between multiplication and division - specifically, in connection with reported use of procedures (Experiment 2). 


\section{Future Directions for the Identical Elements Model}

The RT results of both experiments fit the IE-r model's predictions for positive transfer quite well. The few exceptions involved relatively small effects, and all were plausibly attributed to mediated transfer. Furthermore, the model always correctly predicted the observed direction of differences between transfer conditions. Indeed, there was no feature of the RT results clearly at odds with the model (e.g., transfer between addition and subtraction and equal transfer for subtraction with identical repetition or with an order change). Instead, the results consistently presented the direction of differences predicted by the IE-r model.

The IE-r model (Rickard, 2005) neatly explains facilitative transfer effects in arithmetic fact retrieval, but there are at least two important directions for development of the model. One of these concerns how problems are functionally related to each other in memory and how the network of related facts is processed (i.e., specific assumptions about excitatory and inhibitory processes). The IE model leads one to think of each arithmetic fact as an isolated entity, but it is well established that processing one problem has effects on other, related problems (Ashcraft, 1992, 1995; Campbell, 1987, 1997; Campbell \& Timm, 2000; Manly \& Spoehr, 1999; Phenix \& Campbell, 2004). For example, Phenix and Campbell demonstrated that repeatedly practicing a multiplication problem facilitated retrieval of the correct product, as was expected, but also reduced the accessibility of other multiples of the factors. Their results apparently indicate that practicing arithmetic facts produces retrieval-induced forgetting of related facts, which implicates inhibitory mechanisms (Anderson $\&$ Bell, 2001). The existence of such negative transfer effects is currently outside the scope of the IE model. In fact, retrieval-induced forgetting effects are not explicitly accounted for by any current model of arithmetic memory, although Campbell's (1995) network interference model, which posits both excitatory and inhibitory interactions among problem nodes, provides a possible architecture for explaining such effects.

A second direction for development of the IE-r model concerns mechanisms of arithmetic strategy choice. The participants' use of the reverse association for multiplication to perform division by factoring is consistent with evidence that strategy selection is adapted so as to efficiently exploit features or regularities of the task environment (Lemaire \& Reder, 1999; Schunn, Lovett, \& Reder, 2001). To accommodate evidence that people might use retrieval or procedural strategies for simple arithmetic, the IE-r model distinguishes between asymptotic IE representations and nonasymptotic IE representations. The model predicts transfer effects that reflect IE principles only when memory strength for an IE representation is near asymptote. When memory strength is below asymptote, transfer might be mediated by procedural strategies (e.g., division by factoring). Thus, at present, the model's predictions about strategy choice are based on the strength of the IE representation. Importantly, however, familiarity with problem operands appears to be a major factor in choosing to retrieve, rather than to calculate (Reder \& Ritter, 1992; Schunn, Reder, Nhouyvanisvong, Richards, \& Stroffolino, 1997). For example, Schunn et al. (1997) showed that attempted use of direct retrieval for newly learned arithmetic facts increased with the familiarity of problem operands independently of the availability of the answer. Campbell et al. (2004) showed that direct retrieval was much more likely when simple addition problems appeared in a familiar digit format $(4+8)$ than when they appeared in an unfamiliar written word format (four + eight). These findings suggest that, to explain strategy choice, the IE model needs to incorporate assumptions about how practice affects operand familiarity, in addition to its effects on problem strength.

\section{CONCLUSIONS}

According to the IE model, different arithmetic facts are represented by a common memory node if and only if they are composed of identical elements. This simple principle is useful for understanding facilitative transfer effects in cognitive arithmetic. The model applies neatly to arithmetic facts, but does it apply more broadly to associative memory in general? There is no reason to believe that the IE principles are restricted to arithmetic. Memory for arithmetic facts follows established principles and processes of associative memory; for example, arithmetic memory is organized by semantic relatedness (Ashcraft, 1995) and reveals both retrieval interference and inhibition (Campbell \& Timm, 2000; Phenix \& Campbell, 2004), a variety of priming effects (Campbell, 1991; Meagher \& Campbell, 1995), and retrieval practice effects (Rickard \& Bourne, 1996). Thus, the study of cognitive arithmetic has shown that number fact memory is governed largely by generic memory principles and processes. Given this, it is likely that IE principles studied in the context of arithmetic would apply to combinatorial memory stimuli in any domain.

Nonetheless, it is also clear that arithmetic memory incorporates domain-specific features that make it unique. For example, there is evidence that cognitive arithmetic draws on specialized neural mechanisms that represent numerical magnitude and afford comparisons of magnitude and approximate calculations (Dehaene, Spelke, Pinal, Stanescu, \& Tsivkin, 1999). The magnitude system appears to be intimately integrated in memory for arithmetic facts (Butterworth et al., 2001; Campbell, 1995; Dehaene \& Cohen, 1991). Similarly, the present experiments provided evidence that unique features of the internal structure of multiplication facts (i.e., the reverse association) afford unique types of strategies (i.e., division by factoring) that are not supported for simple addition. Evidence for such domain-specific features in cognitive arithmetic (see also Butterworth et al., 2001; Verguts \& Fias, 2005) gives us new insights into the nature of arithmetic skills 
and brings us closer to a sophisticated understanding of this basic human intellectual ability.

\section{REFERENCES}

Anderson, M. C., \& Bell, T. (2001). Forgetting our facts: The role of inhibitory processes in the loss of propositional knowledge. Journal of Experimental Psychology: General, 130, 544-570.

AshCraft, M. H. (1992). Cognitive arithmetic: A review of theory and data. Cognition, 44, 75-106.

AshCraft, M. H. (1995). Cognitive psychology and simple arithmetic: A review and summary of new directions. Mathematical Cognition, 1, 3-34.

BUtTERWORTH, B. (1999). What counts: How every brain is hardwired for math. New York: Free Press.

Butterworth, B., Zorzi, M., Girelli, L., \& Jonkheere, A. R. (2001). Storage and retrieval of addition facts: The role of number comparison. Quarterly Journal of Experimental Psychology, 54A, 1005-1029.

Campbell, J. I. D. (1987). Network interference and mental multiplication. Journal of Experimental Psychology: Learning, Memory, \& Cognition, 13, 109-123.

CAMpbell, J. I. D. (1991). Conditions of error priming in number-fact retrieval. Memory \& Cognition, 19, 197-209.

Campbell, J. I. D. (1995). Mechanisms of simple addition and multiplication: A modified network-interference theory and simulation. Mathematical Cognition, 1, 121-164.

CAMPBell, J. I. D. (1997). On the relation between skilled performance of simple division and multiplication. Journal of Experimental Psychology: Learning, Memory, \& Cognition, 23, 1140-1159.

CAmpbell, J. I. D. (1999). Division by multiplication. Memory \& Cognition, 27, 791-802.

Campbell, J. I. D., \& Austin, S. (2002). Effects of response time deadlines on adults' strategy choices for simple addition. Memory \& Cognition, 30, 988-994.

Campbell, J. I. D., \& Fugelsang, J. (2001). Strategy choice for arithmetic verification: Effects of numerical surface form. Cognition, $\mathbf{8 0}$, B21-B30.

Campbell, J. I. D., Parker, H. R., \& Doetzel, N. L. (2004). Interactive effects of numerical surface form and operand parity in cognitive arithmetic. Journal of Experimental Psychology: Learning, Memory, \& Cognition, 30, 51-64.

Campbell, J. I. D., \& Timm, J. C. (2000). Adults' strategy choices for simple addition: Effects of retrieval interference. Psychonomic Bulletin \& Review, 7, 692-699.

Campbell, J. I. D., \& Xue, Q. (2001). Cognitive arithmetic across cultures. Journal of Experimental Psychology: General, 130, 299-315.

Cipolotti, L., \& De Lacy Costello, A. (1995). Selective impairment for simple division. Cortex, 31, 433-449.

Cohen, L., Dehaene, S., Chochon, F., Lehericy, S., \& Naccache, L. (2000). Language and calculation within the parietal lobe: A combined cognitive, anatomical, and fMRI study. Neuropsychologia, 38, 1426-1440.

Dehaene, S. (1997). The number sense: How the mind creates mathematics. New York: Oxford University Press.

Dehaene, S., \& Cohen, L. (1991). Two mental calculation systems: A case study of severe acalculia with preserved approximation. Neuropsychologia, 29, 1045-1074.

Dehaene, S., Spelke, E., Pinal, P., Stanescu, R., \& Tsivkin, S. (1999). Sources of mathematical thinking: Behavioral and brainimaging evidence. Science, 284, 970-974.

GEary, D. C. (1996). The problem size effect in mental addition: Developmental and cross-national trends. Mathematical Cognition, 2, 63-93.

Geary, D. C., Frensch, P. A., \& Wiley, J. D. (1993). Simple and complex mental subtraction: Strategy choice and speed-of-processing differences in younger and older adults. Psychology \& Aging, 8, 242-256.

Hecht, S. A. (1999). Individual solution processes while solving addition and multiplication math facts in adults. Memory \& Cognition, 27, 1097-1107.

LeFevre, J., Bisanz, J., Daley, K. E., Buffone, L., Greenham, S. L., \& SADESKY, G. S. (1996). Multiple routes to solution of single-digit multiplication problems. Journal of Experimental Psychology: General, 125, 284-306.

LeFeVre, J., \& Morris, J. (1999). More on the relation between division and multiplication in simple arithmetic: Evidence for mediation of division solutions via multiplication. Journal of Experimental Psychology: Learning, Memory, \& Cognition, 12, 151-175.

LeFevre, J., Sadesky, G. S., \& Bisanz, J. (1996). Selection of procedures in mental addition: Reassessing the problem size effect in adults. Journal of Experimental Psychology: Learning, Memory, \& Cognition, 22, 216-230.

Lemaire, P., \& Reder, L. (1999). What affects strategy selection in arithmetic? The example of parity and five effects on product verification. Memory \& Cognition, 27, 364-382.

Logan, G. D. (1988). Toward an instance theory of automatization. Psychological Review, 95, 492-527.

Manly, C. F., \& Spoehr, K. T. (1999). Mental multiplication: Nothing but the facts? Memory \& Cognition, 27, 1087-1096.

Masson, M. E. J., \& LofTus, G. R. (2003). Using confidence intervals for graphically based data interpretation. Canadian Journal of Experimental Psychology, 57, 203-220.

Mauro, D. G., LeFeVre, J., \& Morris, J. (2003). Effects of format on division and multiplication performance: Division via multiplicationbased representations. Journal of Experimental Psychology: Learning, Memory, \& Cognition, 29, 163-170.

McCloskey, M., Aliminosa, D., \& Sokol, S. M. (1991). Facts, rules, and procedures in normal calculation: Evidence from multiple singlecase studies of impaired arithmetic fact retrieval. Brain \& Cognition, 17, 154-203.

Meagher, P. D., \& Campbell, J. I. D. (1995). Effects of prime type and delay on multiplication priming: Evidence for a dual-process model. Quarterly Journal of Experimental Psychology, 48A, 801-821.

Phenix, T. L., \& CAmpbell, J. I. D. (2004). Effects of multiplication practice on product verification: Integrated structures model or retrieval-induced forgetting? Memory \& Cognition, 32, 324-335.

Reder, L. M., \& RitTer, F. E. (1992). What determines initial feeling of knowing? Familiarity with question terms, not with the answer. Journal of Experimental Psychology: Learning, Memory, \& Cognition, 18, 435-452.

RICKARD, T. C. (1997). Bending the power law: A CMPL theory of strategy shifts and the automatization of cognitive skills. Journal of Experimental Psychology: General, 126, 288-311.

RICKARD, T. C. (2005). A revised identical elements model of arithmetic fact representation. Journal of Experimental Psychology: Learning, Memory, \& Cognition, 31, 250-257.

Rickard, T. C., \& Bourne, L. E., JR. (1996). Some tests of an identical elements model of basic arithmetic skills. Journal of Experimental Psychology: Learning, Memory, \& Cognition, 22, 1281-1295.

Rickard, T. C., Healy, A. F., \& Bourne, L. E., JR. (1994). On the representation of arithmetic facts: Operand order, symbol, and operation transfer effects. Journal of Experimental Psychology: Learning, Memory, \& Cognition, 20, 1139-1153.

Rusconi, E., Galfano, G., Rebonato, E., \& Umiltà, C. (2006). Bidirectional links in the network of multiplication facts. Psychological Research, 70, 32-42.

Schunn, C. D., Lovett, M. C., \& Reder, L. M. (2001). Awareness and working memory in strategy adaptivity. Memory \& Cognition, 29, 254-266.

Schunn, C. D., Reder, L. M., Nhouyvanisvong, A., Richards, D. R. \& Stroffolino, P. J. (1997). To calculate or not to calculate: A source activation confusion model of problem familiarity's role in strategy selection. Journal of Experimental Psychology: Learning, Memory, \& Cognition, 23, 3-29.

Seyler, D. J., Kirk, E. P., \& Ashcraft, M. H. (2003). Elementary subtraction. Journal of Experimental Psychology: Learning, Memory, \& Cognition, 29, 1339-1352.

van Harskamp, N. J., \& Cipolotti, L. (2001). Selective impairments for addition, subtraction, and multiplication: Implications for the organization of arithmetical facts. Cortex, 37, 363-388.

VERGUTS, T., \& FIAS, W. (2005). Interacting neighbors: A connectionist model of retrieval in single-digit multiplication. Memory \& Cognition, 33, 1-16.

Zbrodoff, N. J., \& Logan, G. D. (2005). What everyone finds: The 
problem size effect. In J. I. D. Campbell (Ed.), Handbook of mathematical cognition (pp. 331-346). New York: Psychology Press.

\section{NOTE}

1. Retrieval savings correspond to increased use of retrieval on probe trials, relative to their prime trial baseline. As an alternative approach to analyzing retrieval savings across transfer conditions, we considered a conditional analysis that would compare the rates of probe retrieval given prime retrieval to probe retrieval given prime nonretrieval. This approach was not viable, because it is inevitably confounded by item difficulty effects.

(Manuscript received July 19, 2004;

revision accepted for publication April 6, 2005.) 